\title{
CARATHÉODORY MEASURE OF CYLINDERS
}

\author{
BY \\ GERALD FREILICH
}

1. Introduction. In [5], J. F. Randolph asked whether the generalizations of length and area preserve the relation that area is the product of length by length. Since the relation is preserved for the Lebesgue definitions of length and area, the question takes on interest when the dimension of the measure is less than the dimension of the space in which it is imbedded, for at least one of the components. Randolph obtained partial results using the well-known definition of measure due to Carathéodory and a modification of the definition due to W. Gross.

Subsequently, A. P. Morse and Randolph [4] obtained positive results using Gillespie measure. As a result of the work of A. S. Besicovitch and P. A. P. Moran [1] and the author [2], the question was settled in the negative for Hausdorff measure. Furthermore in [2], the author obtained positive results for Favard measure and negative results for Sphere measure. However, the original question asked by Randolph concerning Carathéodory measure remained unanswered. Roughly speaking, Randolph proved that the Carathéodory area does not exceed the product of length by length, while the reverse inequality, despite some lower bounds obtained by the author in [3], yielded to no method of attack. The present paper settles the question for Carathéodory measure in the negative.

Briefly summarizing our results, in $\$ 3$ below a new definition of area in Euclidean 3-space is introduced, based on horizontal projection only. This is in contrast to Carathéodory's definition which involves projection in all directions; however for cylinder sets, these two definitions agree (Theorem 3.8). This result is applied in $\$ 6$.

In $\$ 4$, a compact subset $A$ of the plane is defined and in $\$ 5$ it is proved that the Carathéodory length of $A$ is greater than 2.22. Then in $\$ 6$ it is proved that the Carathéodory area of the cylinder in 3-space of unit height above $A$ is less than 2.172. Since in Euclidean one-dimensional space, Lebesgue length and Carathéodory length agree, the set $A$ provides an example of a set for which the area of $A \times h$ is strictly less than the length of $A$ multiplied by $h$.

2. Preliminaries. In the remainder of the paper, we shall agree on the following conventions:

If $a>0$, then $a / 0=\infty$.

For any set $X,\{X\}$ will denote the singletonic set whose only element is $X$.

Presented to the Society, March 6, 1963; received by the editors November 1, 1963. 
For real $a,[a]$ will denote the greatest integer in $a$.

$E^{n}$ will denote Euclidean $n$-space. In particular, for $E^{3}$, we shall let $\rho, \theta, \phi$ denote the usual spherical coordinates where $\phi=0$ corresponds to the $z$-axis and $\theta=0, \phi=\pi / 2$ corresponds to the $x$-axis.

For each positive integer $n, L_{n}$ will denote Lebesgue $n$-dimensional measure in $E^{n}$.

For a subset $X$ of the domain of a function $f$, we shall use $f(X)$ to denote $\{y \mid y=f(x)$ for some $x \in X\}$. Also for $A \subset E^{n}, x \in E^{n}$, we let $A+x$ denote $\{a+x \mid a \in A\}$.

Definition 2.1. For $0 \leqq \theta<2 \pi, 0 \leqq \phi<\pi$ we let $p_{\theta, \phi}$ denote the projection of $E^{3}$ in the direction determined by $\theta$ and $\phi$, namely

$$
p_{\theta, \phi}(x, y, z)=(u, v),
$$

where

$$
\begin{aligned}
& u=x\left(\cos \phi \cos ^{2} \theta+\sin ^{2} \theta\right)+y \sin \theta \cos \theta(\cos \phi-1)-z \sin \phi \cos \theta, \\
& v=x \sin \theta \cos \theta(\cos \phi-1)+y\left(\cos \phi \sin ^{2} \theta+\cos ^{2} \theta\right)-z \sin \phi \sin \theta .
\end{aligned}
$$

In terms of $p_{\theta, \phi}$, we define the function $g_{1}$ on the set of all subsets of $E^{3}$ by

$$
g_{1}(X)=\sup _{\theta, \phi} L_{2}\left(p_{\theta, \phi}(X)\right) \quad \text { for } X \subset E^{3} .
$$

Definition 2.2. We shall say that $g$ is a gauge over $E^{n}$ if $g$ is a non-negative function defined over a class $\mathscr{M}$ of subsets of $E^{n}$. We shall say that the (outer) measure $\mu$ is generated by the gauge $g$ if

$\mu(X)=\lim _{r \rightarrow 0+} \inf \left\{\sum_{i=1}^{\infty} g\left(M_{i}\right) \mid X \subset \bigcup_{i=1}^{\infty} M_{i}, \operatorname{diam} M_{i}<r, M_{i} \in \mathscr{M}\right\}$ for $X \subset E^{n}$.

It is easy to see that if two sets are a positive distance apart, then the $\mu$-measure of the union is the sum of the individual $\mu$-measures and from this it follows that all Borel sets are $\mu$-measurable.

Definition 2.3. For $k$ and $n$ positive integers with $k \leqq n$, let $g$ be defined on the set of all convex open subsets of $E^{n}$ by the formula

$$
g(X)=\sup \left\{L_{k}(P(X)) \mid P \text { is an orthogonal projection of } E^{n} \text { onto } E^{k}\right\} .
$$

(Note that when $k=2, n=3$, then $g$ agrees with the function $g_{1}$ defined in 2.1 , while for $k=1$ and arbitrary $n, g(X)$ is the diameter of $X$.)

We define the Carathéodory $k$-dimensional measure in $E^{n}$, denoted by $C_{n}^{k}$, as the measure generated by the gauge $g$. In particular, note that $C_{n}^{n}=L_{n}$. Also if $m<n$ and we think of $E^{m}$ as imbedded in $E^{n}$, then for $k \leqq m$ and $X \subset E^{m}$ it is easily seen that $C_{m}^{k}(X)=C_{n}^{k}(X)$.

\section{A characterization of $C_{3}^{2}$-measure of cylinders.}

DEFinition 3.1. Let $g_{2}$ be the function defined for all open convex subsets 
$X$ of $E^{3}$ by

$$
g_{2}(X)=\sup _{\theta} L_{2}\left(p_{\theta, \pi / 2}(X)\right) .
$$

Definition 3.2. Let $\lambda \geqq 0$ and define $\mathscr{M}_{\lambda}$ to be the set of all open convex subsets $X$ of $E^{3}$ such that

$$
g_{2}(X) \geqq \lambda \cdot L_{2}\left(p_{\theta, 0}(X)\right) .
$$

Then $D_{\lambda}$ is defined to be the measure generated by the gauge $g_{2}$ restricted to the sets in $\mathscr{M}_{\lambda}$.

Definition 3.3. A subset $Z$ of $E^{3}$ will be called a cylinder if and only if $Z=X \times I$ where $X \subset E^{2}$ and $I$ is an interval of real numbers of positive length.

THEOREM 3.4. If $\lambda>\mu>0$, then for arbitrary $X \subset E^{3}$,

$$
D_{\lambda}(X) \geqq D_{\mu}(X)
$$

while if $Z \subset E^{3}$ is a cylinder,

$$
D_{\lambda}(Z)=D_{\mu}(Z) .
$$

Proof. Since $\lambda>\mu$, it follows that $\mathscr{M}_{\lambda} \subset \mathscr{M}_{\mu}$ (using the terminology of Definition 3.2). Hence from the definition of the measure generated by a gauge, it follows that $D_{\mu}(X) \leqq D_{\lambda}(X)$ for any $X \subset E^{3}$.

Next, if $Z$ is a cylinder, we may assume without loss of generality that $Z=X \times[0,1]$ where $X \subset E^{2}$. Using the invariance of $D_{\lambda}$ under translation and 2.2, it is easy to see that, irrespective of the $D_{\lambda}$-measurability of $Z$,

$$
D_{\lambda}(X \times[0, k])=k \cdot D_{\lambda}(Z) \quad \text { for } k>0 ;
$$

similarly

$$
D_{\mu}(X \times[0, k])=k \cdot D_{\mu}(Z) .
$$

Let $k=\lambda / \mu$ and $T$ be the transformation from $E^{3}$ to $E^{3}$ defined by $T(x, y, z)$ $=(x, y, k z)$. Then $U \in \mathscr{M}_{\mu}$ implies that $T(U) \in \mathscr{M}_{\lambda}, g_{2}(T(U))=k \cdot g_{2}(U)$, and $\operatorname{diam} T(U) \leqq k \cdot \operatorname{diam} U$. Now let $\epsilon>0$ be arbitrary, and choose a countable covering of $Z$ by sets $U_{i} \in \mathscr{M}_{\mu}$ with $\operatorname{diam} U_{i}<\epsilon / k$ and

$$
\sum_{i} g_{2}\left(U_{i}\right)<D_{\mu}(Z)+\epsilon / k .
$$

Then the sets $T\left(U_{i}\right) \in \mathscr{M}_{\lambda}$ form a countable covering of $T(Z)=X \times[0, k]$, with $\operatorname{diam} T\left(U_{i}\right)<\epsilon$ and

$$
\sum_{i} g_{2}\left(T\left(U_{i}\right)\right)=k \cdot \sum_{i} g_{2}\left(U_{i}\right)<k \cdot D_{\mu}(Z)+\epsilon .
$$

Since $\epsilon$ was arbitrary, 


$$
D_{\lambda}(T(Z))=k \cdot D_{\lambda}(Z) \leqq k \cdot D_{\mu}(Z),
$$

or

$$
D_{\lambda}(Z) \leqq D_{\mu}(Z) \text {. }
$$

Combining with the first part of the proof yields

$$
D_{\lambda}(Z)=D_{\mu}(Z) \text {. }
$$

Example 3.5. If $X=\{(x, y, z) \mid 0 \leqq x \leqq 1,0 \leqq y \leqq 1, z=0\}$, then it is easy to prove that $D_{\lambda}(X)=\lambda$, so that $\lambda \neq \mu$ implies that $D_{\lambda} \not \equiv D_{\mu}$.

Lemma 3.6. Let $X$ be a convex subset of $E^{3}, \quad X_{1}=p_{0, \pi / 2}(X), X_{2}=p_{0,0}(X)$, $X_{3}=p_{\theta, \phi}(X)$ where $0 \leqq \phi \leqq \pi$. Then

$$
L_{2}\left(X_{3}\right) \leqq L_{2}\left(X_{1}\right) \cdot \sin \phi+L_{2}\left(X_{2}\right) \cdot|\cos \phi| \text {. }
$$

Proof. Without loss of generality, we may take $\theta=\pi / 2$. Let $Y=p_{\theta, \pi / 2}^{-1}\left(X_{1}\right)$ $\cap p_{\theta, 0}^{-1}\left(X_{2}\right)$. Then $Y$ is convex and $X \subset Y$. Also

$$
\begin{aligned}
L_{1}\left(p_{\theta, \phi}(Y) \cap\{(x, y) \mid x=t\}\right)= & L_{1}\left(X_{1} \cap\{(x, y) \mid x=t\}\right) \cdot \sin \phi \\
& +L_{1}\left(X_{2} \cap\{(x, y) \mid x=t\}\right) \cdot|\cos \phi| .
\end{aligned}
$$

Then

$$
\begin{aligned}
L_{2}\left(X_{3}\right) \leqq & L_{2}\left(p_{\theta, \phi}(Y)\right)=\int_{-\infty}^{\infty} L_{1}\left(p_{\theta, \phi}(Y) \cap\{(x, y) \mid x=t\}\right) d t \\
= & \sin \phi \cdot \int_{-\infty}^{\infty} L_{1}\left(X_{1} \cap\{(x, y) \mid x=t\}\right) d t \\
& \quad+|\cos \phi| \cdot \int_{-\infty}^{\infty} L_{1}\left(X_{2} \cap\{(x, y) \mid x=t\}\right) d t \\
= & \sin \phi \cdot L_{2}\left(X_{1}\right)+|\cos \phi| \cdot L_{2}\left(X_{2}\right) .
\end{aligned}
$$

Lemma 3.7. Let $X$ be a bounded open convex subset of $E^{3}$. Then $X$ is contained in an open convex set $Y$ such that

$$
\operatorname{diam} Y=\operatorname{diam} X, \quad g_{1}(Y)=g_{1}(X)
$$

and

$$
g_{2}(Y) \geqq 10^{-2} \cdot L_{2}\left(p_{\theta, 0}(Y)\right) .
$$

Proof. Let $X_{1}=p_{\theta, 0}(X), \bar{X}_{1}$ be the closure of $X_{1}$ in $E^{2}, \bar{X}$ the closure of $X$ in $E^{3}$. We may suppose that

$$
0<g_{2}(X)<10^{-2} \cdot L_{2}\left(X_{1}\right),
$$

for otherwise we may take $X=Y$. Let $d_{1}$ be the diameter of $X_{1}$ and by subjecting the $x-y$ plane to a rigid motion if necessary, we may choose points $\left(0,0, z_{1}\right) \in \bar{X},\left(d_{1}, 0, z_{2}\right) \in \bar{X}$. Let 


$$
d_{2}=\sup _{x} L_{1}\left(\left\{t \mid(x, t) \in X_{1}\right\}\right) .
$$

First we observe that $\left|z_{1}-z_{2}\right|<d_{1} / 50$. For if $\left|z_{1}-z_{2}\right| \geqq d_{1} / 50$, then $g_{2}(X) \geqq L_{2}\left(p_{0, \mathbf{x} / 2}(X)\right) \geqq \frac{1}{2} d_{2} \cdot d_{1} / 50=10^{-2}\left(d_{1} d_{2}\right) \geqq 10^{-2} L_{2}\left(X_{1}\right)$, a contradiction.

Next let $0 \leqq \psi<\pi$ be such that $\tan \psi=d_{1} /\left(z_{1}-z_{2}\right)$ and let

$$
\begin{aligned}
& x \cos \psi+z \sin \psi=p_{1}, \\
& x \cos \psi+z \sin \psi=p_{2}, \quad p_{2}>p_{1},
\end{aligned}
$$

be two support planes to $X$. Then $|\tan \psi| \geqq 50$ and $p_{2}-p_{1}<d_{2} / 50$, for if $p_{2}-p_{1} \geqq d_{2} / 50$, then

$$
g_{2}(X) \geqq L_{2}\left(p_{\pi / 2, \pi / 2}(X)\right) \geqq \frac{1}{2}\left(d_{2} d_{1}\right) / 50=10^{-2}\left(d_{1} d_{2}\right) \geqq 10^{-2} L_{2}\left(X_{1}\right) .
$$

Define $y_{1}=\sup \left\{t \mid\left(d_{1} / 2, t\right) \in X_{1}\right\} \geqq 0, \quad y_{2}=\inf \left\{t \mid\left(d_{1} / 2, t\right) \in X_{1}\right\} \leqq 0$, and $y_{3}=\left(y_{1}+y_{2}\right) / 2$. Then $y_{2}-y_{1} \geqq d_{2} / 2$. Since $d_{1} \geqq d_{2}$, it follows that the circumference $W$ with center at $\left(d_{1} / 2, y_{3}\right)$ and radius $d_{2} \sqrt{ } 2 / 8$ is contained within $\bar{X}_{1}$. Define

$$
\begin{gathered}
z_{1}=\sup \left\{t \mid\left(d_{1} / 2, y_{3}, t\right) \in X\right\}, z_{2}=\inf \left\{t \mid\left(d_{1} / 2, y_{3}, t\right) \in X\right\}, \\
z_{3}=\left(z_{1}+z_{2}\right) / 2,
\end{gathered}
$$

and $h>0$ to be the number such that the closed convex set $\bar{Y}$ in $E^{3}$ generated by $\bar{X}$ and the points $\left(d_{1} / 2, y_{3}, z_{3}-h\right),\left(d_{1} / 2, y_{3}, z_{3}+h\right)$ has the property that $g_{2}(\bar{Y})=10^{-2} \cdot L_{2}\left(X_{1}\right)$. Then $h \leqq d_{2} / 100$, for if not,

$$
g_{2}(\bar{Y}) \geqq L_{2}\left(p_{\pi / 2, \pi / 2}(Y)\right)>\left(\frac{1}{2}\left(2 d_{2}\right) / 100\right) \cdot d_{1} \geqq 10^{-2} L_{2}\left(X_{1}\right) .
$$

Now let $(x, y, z) \in \bar{X}$ with $(x, y) \in W$. Then

$$
\begin{aligned}
\left|z_{3} \pm h-z\right| & \leqq h+\left(p_{2}-p_{1}\right) \cdot|\csc \psi|+\left(d_{2} \sqrt{ } 2 / 8\right) \cdot|\cot \psi| \\
& \leqq\left(d_{2} / 100\right)+\left(d_{2} / 50\right)(\sqrt{ }(2501) / 50)+\left(d_{2} \sqrt{ } 2 / 8\right)(1 / 50) \\
& <d_{2} / 20
\end{aligned}
$$

so that the absolute value of the tangent of the angle of elevation from $(x, y, z)$ to $\left(d_{1} / 2, y_{3}, z_{3} \pm h\right)$ is less than $\left(d_{2} / 20\right) /\left(d_{2} \sqrt{ } 2 / 8\right)=\sqrt{ } 2 / 5$. Hence for $0 \leqq \phi$ $\leqq \tan ^{-1} 5 / \sqrt{ } 2$ and for all $\theta$, it follows that $p_{\theta, \phi}(\bar{Y})=p_{\theta, \phi}(\bar{X})$.

For $\tan ^{-1} 5 / \sqrt{ } 2 \leqq \phi \leqq \pi / 2$ and for all $\theta$, we have by use of Lemma 3.3,

$$
\begin{aligned}
L_{2}\left(p_{\theta, \phi}(\bar{Y})\right) & \leqq L_{2}\left(p_{\theta, 0}(\bar{Y})\right) \cdot \cos \phi+L_{2}\left(p_{\theta, \pi / 2}(\bar{Y})\right) \cdot \sin \phi \\
& \leqq L_{2}\left(\bar{X}_{1}\right)(\sqrt{ } 2 / \sqrt{ }(27))+10^{-2} \cdot L_{2}\left(\bar{X}_{1}\right) \\
& <L_{2}\left(X_{1}\right) .
\end{aligned}
$$

Hence $g_{1}(\bar{Y})=g_{1}(\bar{X})=g_{1}(X)$.

If we define $Y$ to be the interior of $\bar{Y}$, then $Y$ is open and convex, $X \subset Y$, $g_{1}(Y)=g_{1}(X)$ and $g_{2}(Y)=10^{-2} L_{2}\left(X_{1}\right)=10^{-2} L_{2}\left(p_{\theta, 0}(Y)\right)$. It is easy to check that $\operatorname{diam} Y=\operatorname{diam} X$. 
TheOREM 3.8. If $\lambda>0$ and $Z$ is a cylinder, then

$$
D_{\lambda}(Z)=C_{3}^{2}(Z) .
$$

Moreover, if $0<\lambda \leqq 10^{-2}$, and $X$ is an arbitrary subset of $E^{3}$, then

$$
D_{\lambda}(X) \leqq C_{3}^{2}(X) \text {. }
$$

Proof. Let us first assume that $0<\lambda \leqq 10^{-2}$, and $X \subset E^{3}$. Let $\epsilon>0$, and choose a countable covering of $X$ by open convex sets $U_{i}$ with $\operatorname{diam} U_{i}<\epsilon$ and $\sum_{i} g_{1}\left(U_{i}\right)<C_{3}^{2}(X)+\epsilon$. By Lemma 3.7 , we may replace each $U_{i}$ by a set $V_{i} \in \mathscr{M}_{\lambda}$ satisfying $U_{i} \subset V_{i}, \operatorname{diam} U_{i}=\operatorname{diam} V_{i}$ and $g_{1}\left(U_{i}\right)=g_{1}\left(V_{i}\right)$. Then the sets $V_{i}$ form a countable covering of $X$ by sets of $\mathscr{M}_{\lambda}$ with diameters less than $\epsilon$ and

$$
\sum_{i} g_{2}\left(V_{i}\right) \leqq \sum_{i} g_{1}\left(V_{i}\right)=\sum_{i} g_{1}\left(U_{i}\right)<C_{3}^{2}(X)+\epsilon .
$$

Hence $D_{\lambda}(X) \leqq C_{3}^{2}(X)$, since $\epsilon$ was arbitrary.

If $0<\lambda \leqq 10^{-2}$ and $Z$ is a cylinder, then without loss of generality we may assume that $Z=Y \times[0,1]$ where $Y \subset E^{2}$. As in Theorem 3.4,

$$
D_{\lambda}(Y \times[0, k])=k \cdot D_{\lambda}(Z)
$$

and

$$
C_{3}^{2}(Y \times[0, k])=k \cdot C_{3}^{2}(Z) \quad \text { for } k>0 .
$$

Let $k>0$ and $\epsilon>0$ be arbitrary and choose a countable covering of $Z$ by sets $U_{i} \in \mathscr{M}_{\lambda}$ with $\operatorname{diam} U_{i}<\epsilon / k$ and

$$
\sum_{i} g_{2}\left(U_{i}\right)<D_{\lambda}(Z)+1 / k
$$

Defining $T(x, y, z)=(x, y, k z)$, it follows that the collection of sets $T\left(U_{i}\right)$ is a countable covering of $Y \times[0, k]$ by open convex sets with diameters no greater than $\epsilon$. By Lemma 3.6,

$$
\begin{aligned}
g_{1}\left(T\left(U_{i}\right)\right) & \leqq \max _{\phi}(|\cos \phi| / \lambda+k \sin \phi) \cdot g_{2}\left(U_{i}\right) \\
& =\left(\left(1+\lambda^{2} k^{2}\right)^{1 / 2} / \lambda\right) \cdot g_{2}\left(U_{i}\right),
\end{aligned}
$$

so that

$$
\begin{aligned}
\sum_{i} g_{1}\left(T\left(U_{i}\right)\right) & \leqq\left(\left(1+\lambda^{2} k^{2}\right)^{1 / 2} / \lambda\right) \sum_{i} g_{2}\left(U_{i}\right) \\
& <\left(\left(1+\lambda^{2} k^{2}\right)^{1 / 2} / \lambda\right) \cdot D_{\lambda}(Z)+\left(1+\lambda^{2} k^{2}\right)^{1 / 2} / \lambda k
\end{aligned}
$$

Since $\epsilon$ was arbitrary, it follows that

$$
C_{3}^{2}(Y \times[0, k])=k \cdot C_{3}^{2}(Z) \leqq\left(\left(1+\lambda^{2} k^{2}\right)^{1 / 2} / \lambda\right) D_{\lambda}(Z)+\left(1+\lambda^{2} k^{2}\right)^{1 / 2} / \lambda k .
$$

Hence 


$$
C_{3}^{2}(Z) \leqq\left(\left(1+\lambda^{2} k^{2}\right)^{1 / 2} / \lambda k\right) D_{\lambda}(Z)+\left(1+\lambda^{2} k^{2}\right)^{1 / 2} / \lambda k^{2},
$$

and letting $k \rightarrow \infty$ yields

$$
C_{3}^{2}(Z) \leqq D_{\lambda}(Z) .
$$

Combining with the fact that $D_{\lambda}(Z) \leqq C_{3}^{2}(Z)$ we conclude that if $0<\lambda \leqq 10^{-2}$, then $D_{\lambda}(Z)=C_{3}^{2}(Z)$. By Theorem 3.4, it follows that for arbitrary $\lambda>0$,

$$
D_{\lambda}(Z)=C_{3}^{2}(Z) \text {. }
$$

REMARK 3.9. If $\lambda=0$, the measure $D_{0}$ obtained is smaller than all $D_{\lambda}$ $(\lambda>0)$ and also $C_{3}^{2}$. An interesting question whose answer is unknown to the author is: If $Z$ is a cylinder, does it necessarily follow that $D_{0}(Z)=C_{3}^{2}(Z)$ ?

4. The construction of the set $A$. The operation $S$ to be defined will be applicable to any collection of rectangles in the plane whose dimensions are in the ratio 2:1, with the longer edge horizontal. Preliminary to the definition we define certain constants.

DEFinition 4.1. Let

$$
\begin{aligned}
& h_{n}=(9027+4.5 n) \cdot 10^{-6} \text { for } n=1,2, \cdots, 9, \\
& h_{n}=\left((447,212+2 n)^{2} \cdot 5 \cdot 10^{-12}-1\right)^{1 / 2} \text { for } n=10 \text { through } 276,390,
\end{aligned}
$$

and let

$$
\begin{array}{ll}
h_{n}=2-(15.5) 10^{-6} & \text { for } n=276,391, \\
h_{n}=2-11 \cdot 10^{-6} & \text { for } n=276,392, \\
h_{n}=2-(6.5) \cdot 10^{-6} & \text { for } n=276,393, \\
h_{n}=2-2 \cdot 10^{-6} & \text { for } n=276,394 .
\end{array}
$$

Let $v=(223,605)^{-1} \cdot\left(1-2 \cdot 10^{-6}\right)$ and $h=102 \cdot 10^{-6}$.

Definition 4.2. Let $R$ be a closed rectangle in the plane of dimensions $q$ by $2 q$, with the side of length $2 q$ horizontal. Let $R_{p}$ denote the unique closed rectangle of dimensions $10^{-6} \cdot q$ by $2 \cdot 10^{-6} \cdot q$ with the longer side horizontal and with the lower left vertices of $R$ and $R_{p}$ coinciding. Define

$$
\begin{aligned}
& R_{2 n}=R_{p}+\left(q \cdot h_{n}, 0\right) \\
& \left.R_{2 n-1}=R_{p}+\left(q \cdot h_{n},\left(1-10^{-6}\right) q\right)\right\} \quad \text { for } n=1, \cdots, n=276,394 \text {, } \\
& R_{n}=R_{p}+(0,(n-552,789) q v) \quad \text { for } n=552,789 \text { through } n=776,394 \text {, } \\
& R_{n}=R_{p}+(q h,(n-776,395) q v) \quad \text { for } n=776,395 \text { through } n=10^{6} \text {, } \\
& \text { and } S(\{R\})=\bigcup_{n=1}^{106}\left\{R_{n}\right\}, S^{*}(\{R\})=\bigcup_{n=1}^{106} R_{n} \text {. }
\end{aligned}
$$

We extend the definitions to collections of rectangles in an obvious manner, that is,

$$
S\left(\bigcup_{i}\left\{R_{i}\right\}\right)=\bigcup_{i} S\left(\left\{R_{i}\right\}\right) \quad \text { and } \quad S^{*}\left(\bigcup_{i}\left\{R_{i}\right\}\right)=\bigcup_{i} S^{*}\left(\left\{R_{i}\right\}\right) .
$$


Definition 4.3. Let $A_{0}^{*}=\{(x, y) \mid 0 \leqq x \leqq 2,0 \leqq y \leqq 1\}, A_{0}=\left\{A_{0}^{*}\right\}$ and define inductively for $i=1,2, \cdots$,

$$
A_{i}=S\left(A_{i-1}\right), \quad A_{i}^{*}=S^{*}\left(A_{i-1}\right) .
$$

Define $A=\bigcap_{i=1}^{\infty} A_{i}^{*}$.

\section{The Carathéodory 1-measure of $A$.}

Theorem 5.1. $C_{2}^{1}(A) \leqq \sqrt{ } 5$.

Proof. Using the notation of Definition 4.3, it is easy to show by induction that at the $i$ th stage of construction, the set $A$ is covered by a collection of $10^{6 i}$ rectangles each of diameter $\sqrt{ } 5 \cdot 10^{-6 i}$ so that

$$
\sum_{a \in A_{i}} \operatorname{diam} a=\sqrt{ } 5, \quad A \subset \bigcup_{a \in A_{i}} a .
$$

Let $\lambda>1$ be arbitrary. Then each $a \in A_{i}$ can be covered by an open rectangle $\hat{a}$ such that $\operatorname{diam} \hat{a}=\lambda \cdot \operatorname{diam} a$. Hence $A \subset \bigcup_{\hat{a}}, \sum \operatorname{diam} \hat{a}=\lambda \sqrt{ } 5$, so that $C_{2}^{1}(A) \leqq \sqrt{ } 5 \lambda$. Let $\lambda \rightarrow 1$ to obtain

$$
C_{2}^{1}(A) \leqq \sqrt{ } 5 .
$$

Definition 5.2. For $X$ an open convex subset of the plane of diameter $d>0$, we define

$$
r(X)=\limsup _{i \rightarrow \infty}\left(d \cdot 10^{6 i}\right) /\left(N_{i} \sqrt{ } 5\right)
$$

where $N_{i}$ is the number of rectangles of $A_{i}$ that have nonvacuous intersection with $X$.

Theorem 5.3. In the notation of Definition 5.2, as $i \rightarrow \infty$,

$$
\frac{d \cdot 10^{6 i}}{N_{i} \sqrt{ } 5} r(X)
$$

Proof. For $R \in A_{i}, R^{\prime} \in S(\{R\})$, then $R \cap X=\emptyset$ implies that $R^{\prime} \cap X=\emptyset$, so that $N_{i+1} \leqq N_{i} \cdot 10^{6}$.

TheOREM 5.4. If $X$ is an open nonvacuous convex subset of the plane, then $r(X) \geqq 0.9932$.

Proof. Let $d>0$ denote the diameter of $X$. Choose $i$ so that $i=1$ if $d>\sqrt{ } 5 \cdot 10^{-6}$ and otherwise choose $i$ so that

$$
\sqrt{ } 5 \cdot 10^{-6 i}<d \leqq \sqrt{ } 5 \cdot 10^{-6 i+6} .
$$

Since the argument for arbitrary $i$ is identical to that for $i=1$ (except for a scale factor which cancels out in the ratio $r(X)$ ), we shall assume that $\sqrt{ } 5 \cdot 10^{-6}<d \leqq \sqrt{ } 5$ and divide the proof into the following four parts. We shall use the notation of Definition 4.2, with $R$ interpreted as $A_{0}^{*}$. In addition, we shall describe those $R_{2 n}$ where $n$ runs from 1 to 276,394 as row $\alpha$, while row 
$\beta$ will consist of $R_{2 n-1}$ with $n$ running from 1 to 276,394 . Column $\gamma$ will consist of those $R_{n}$ with $n$ between 552,789 and 776,394, while column $\delta$ will consist of those $R_{n}$ with $n$ between 776,395 and 1,000,000.

PART 1. If $\sqrt{ } 5 \cdot 10^{-6}<d \leqq 2 \sqrt{ } 5 \cdot 10^{-6}$, then $r(X) \geqq 1$.

Proof. Note that $\left|h_{i}-h_{j}\right| \geqq 4.5 \cdot 10^{-6}>2 \sqrt{ } 5 \cdot 10^{-6}+2 \cdot 10^{-12}$ for $i \neq j$. Since $v>2 \sqrt{ } 5 \cdot 10^{-6}+2 \cdot 10^{-12}$, it follows that $X$ can have nonvacuous intersection with at most two $R_{i}$ and these $R_{i}$ must be of the following form:

(a) Either they are two consecutive rectangles in row $\alpha$ or in row $\beta$,

(b) or they are two consecutive rectangles in column $\gamma$ or in column $\delta$. In case (a), consider for example $R_{2 n}$ and $R_{2 n+2}$. Since the vertical support lines of the convex set $X$ are at a distance no greater than $d \leqq 2 \sqrt{ } 5 \cdot 10^{-6}$ while $R_{2 n+2}$ is a horizontal translate of $R_{2 n}$ by an amount greater than $2 \sqrt{ } 5 \cdot 10^{-6}+2 \cdot 10^{-12}$, it follows that $X$ cannot intersect more than $10^{6}$ rectangles in $S\left(\left\{R_{2 n}\right\}\right) \cup S\left(\left\{R_{2 n+2}\right\}\right)$, so that $N_{2} \leqq 10^{6}$. Hence applying Theorem 5.3,

$$
r(X) \geqq \frac{d \cdot 10^{12}}{N_{2} \sqrt{ } 5}>\frac{\sqrt{ } 5 \cdot 10^{-6} \cdot 10^{12}}{10^{6} \sqrt{ } 5}=1
$$

In case (b), the same considerations apply except that the horizontal support lines of $X$ are considered instead of the vertical. Finally if $X$ intersects less than two $R_{i}$, it is obvious that $r(X) \geqq 1$.

PART 2. If $2 \sqrt{ } 5 \cdot 10^{-6}<d<100 \cdot 10^{-6}$, then $r(X) \geqq 1$.

Proof. Because $h_{1}>h=102 \cdot 10^{-6}$, it follows that $X$ can have nonvacuous intersection with a sequence of $R_{i}$ which must be of one of the following forms:

(a) Either they are a consecutive sequence of rectangles of row $\alpha$ or of row $\beta$,

(b) or they form a consecutive sequence of rectangles of column $\gamma$ or of column $\delta$.

In either case, if $X$ intersects two or less of the $R_{i}$, then

$$
r(X) \geqq \frac{2 \sqrt{ } 5 \cdot 10^{-6} \cdot 10^{6}}{2 \sqrt{ } 5}=1 .
$$

In case (a), if $X$ intersects $n>2$ of the $R_{i}$ nonvacuously, then $d>(n-2)$ $\cdot(4.5) 10^{-6}+(2.5) 10^{-6}$. Hence

$$
\begin{aligned}
r(X) & \geqq \frac{\left((n-2)(4.5) 10^{-6}+(2.5) 10^{-6}\right) \cdot 10^{6}}{n \sqrt{ } 5} \\
& =\frac{(n-2)(4.5)+2.5}{n \sqrt{ } 5}>1
\end{aligned}
$$


In case (b), if $X$ intersects $n>2$ of the $R_{i}$, then

$$
d>(n-1) v-10^{-6},
$$

so that

$$
r(X) \geqq \frac{\left((n-1) v-10^{-6}\right) \cdot 10^{6}}{n \sqrt{ } 5}>\frac{(n-1) 2 \sqrt{ } 5-1}{n \sqrt{ } 5}>1 .
$$

PART 3. If $100 \cdot 10^{-6} \leqq d \leqq 8927 \cdot 10^{-6}$, then $r(X)>0.996$.

Proof. Taking into account the sizes of $h_{1}$ and $h$, it is obvious that $X$ cannot intersect nonvacuously a rectangle from either row $\alpha$ or row $\beta$ simultaneously with a rectangle from column $\gamma$ or column $\delta$. We shall assume that $X$ does not intersect either row $\alpha$ or row $\beta$, for if it did, the argument of Part 2 would imply that $r(X)>1$.

Without loss of generality, we assume that $X$ intersects column $\gamma$ in $j>2$ rectangles and column $\delta$ in no more than $j$ rectangles. Hence $d$ must be greater than $(j-1) v-10^{-6}$. We divide the remainder of the proof into three cases.

Case 1. $X$ intersects column $\delta$ in less than $(j-2)$ rectangles. In this case,

$$
r(X) \geqq \frac{d \cdot 10^{6}}{(2 j-3) \sqrt{ } 5} \geqq \frac{(j-1) v \cdot 10^{6}-1}{(2 j-3) \sqrt{ } 5} \geqq \frac{(j-1) 2 \sqrt{ } 5-1}{(2 j-3) \sqrt{ } 5}>1 .
$$

Case 2. $X$ intersects column $\delta$ in exactly $(j-2)$ rectangles. In this case, since columns $\gamma$ and $\delta$ are separated by a distance of $10^{-4}$, it follows that

$$
d>\left(\left(10^{-4}\right)^{2}+\left((j-2) v-10^{-6}\right)^{2}\right)^{1 / 2} .
$$

A little algebra shows that if $j \leqq 202$, the right member is greater than $(j-1) v$, so that $r(X)>(j-1) v \cdot 10^{6} /(2 j-2) \sqrt{ } 5>1$. If $j>202$, then as above $d>(j-1) v-10^{-6}$ so that

$$
r(X)>\frac{\left((j-1) v-10^{-6}\right) 10^{6}}{(2 j-2) \sqrt{ } 5}>\frac{2(j-1) \sqrt{ } 5-1}{2(j-1) \sqrt{ } 5}>.998 .
$$

Case 3. $X$ intersects column $\delta$ in more than $(j-2)$ rectangles. In this case, $d>\left(\left(10^{-4}\right)^{2}+\left((j-1) v-10^{-6}\right)^{2}\right)^{1 / 2}$. By Case 2 , if $j \leqq 201$, then $d>j v$ so that $r(X)>j v \cdot 10^{-6} /(2 j \sqrt{ } 5)>1$; while if $201<j<345$, then

$$
\left(\left(10^{-4}\right)^{2}+\left((j-1) v-10^{-6}\right)^{2}\right)^{1 / 2} \geqq\left(j-\frac{1}{2}\right) v
$$

so that

$$
r(X)>\frac{(2 j-1) \sqrt{ } 5}{2 j \sqrt{ } 5}=1-(1 / 2 j)>.997
$$

finally if $j \geqq 345$, then

$$
r(X)>\frac{\left((j-1) v-10^{-6}\right) 10^{6}}{2 j \sqrt{ } 5}>\frac{2(j-1) \sqrt{ } 5-1}{2 j \sqrt{ } 5}>.996 .
$$


PART 4. If $8927 \cdot 10^{-6}<d \leqq \sqrt{ } 5$, then $r(X)>.9932$.

Proof. If $X$ intersects only row $\alpha$ (or row $\beta$ ), the argument of Part 2 implies that $r(X) \geqq 1$. If $X$ intersects neither row $\alpha$ nor row $\beta$, the last argument of Case 3, Part 3 implies that $r(X)>0.996$. The remainder of the proof is divided into 3 cases.

Case 1. The intersection of $X$ and $A_{0}^{*}$ is contained in rows $\alpha$ and $\beta$.

In view of what has been proved already, we may assume that $X$ meets row $\alpha$ in $N_{\alpha}>0$ rectangles and row $\beta$ in $N_{\beta}>0$ rectangles, and that $N_{\alpha} \geqq N_{\beta}$. In fact, we may take $N_{\alpha}>1$. Since $h_{n+1}-h_{n} \geqq(4.5) \cdot 10^{-6}$, it follows that there exists a point common to row $\alpha$ and $X$ and a point common to row $\beta$ and $X$ such that the absolute value of the difference of abscissas is no less than

$$
\frac{1}{2}\left(\left(N_{\alpha}+N_{\beta}-2\right)(4.5) \cdot 10^{-6}-4 \cdot 10^{-6}\right) \geqq \frac{1}{2}\left(N_{\alpha}+N_{\beta}-3\right)(4.5) \cdot 10^{-6} .
$$

Since the distance from row $\alpha$ to row $\beta$ is $1-2 \cdot 10^{-6}$, it follows that

$$
\begin{aligned}
d & \geqq\left(\left(1-2 \cdot 10^{-6}\right)^{2}+\frac{1}{4}\left(\left(N_{\alpha}+N_{\beta}-3\right)(4.5) 10^{-6}\right)^{2}\right)^{1 / 2} \\
& \geqq\left(1-2 \cdot 10^{-6}\right)\left(1+5\left(N_{\alpha}+N_{\beta}-3\right)^{2} 10^{-12}\right)^{1 / 2} .
\end{aligned}
$$

Hence

$$
r(X) \geqq \frac{\left(10^{6}-2\right)\left(1+5\left(N_{\alpha}+N_{\beta}-3\right)^{2} 10^{-12}\right)^{1 / 2}}{\left(N_{\alpha}+N_{\beta}\right) \sqrt{ } 5}>1,
$$

since $N_{\alpha}+N_{\beta} \leqq 552,788$.

Case 2. $X$ meets only one of the two rows $\alpha$ and $\beta$.

Again we may assume that $X$ does not meet row $\beta$, but does intersect row $\alpha$ and at least one of the two columns $\gamma$ and $\delta$. Let $N_{v}+1$ be the smallest number $n$ such that $X$ meets no rectangle above the horizontal line $y=n v+10^{-6}$, and let $N$ be the total number of rectangles $R_{i}$ that intersect $X$ nonvacuously. If $N \leqq 3990$, then since $d>8927 \cdot 10^{-6}$ it would follow that $r(X)>1$. Hence we shall assume that $N>3990$. Also, we may take $N_{v} \geqq 0$.

Let $M$ be the largest value of $n \leqq 276,394$ such that $h_{n} \leqq\left(d^{2}-v^{2} N_{v}^{2}\right)^{1 / 2}$. Without loss of generality, we may assume $M \geqq 1$. Then at most $M$ $+\left[106 \cdot 10^{-6} /(4.5) \cdot 10^{-6}\right]=M+23$ of the rectangles of row $\alpha$ can intersect $X$. Hence $N \leqq 2\left(N_{v}+2\right)+M+23=2 N_{v}+M+27$, so that

$$
\frac{2 N_{v}+M}{N} \geqq \frac{N-27}{N} \geqq 1-(27 / 3990)>.9932 \text {. }
$$

Now

$$
r(X)=\frac{d \cdot 10^{6}}{N \sqrt{ } 5} \geqq(.9932) \frac{d \cdot 10^{6}}{\left(2 N_{v}+M\right) \sqrt{ } 5} \geqq(.9932) \frac{\left(h_{M}^{2}+v^{2} N_{v}^{2}\right)^{1 / 2} \cdot 10^{6}}{\left(2 N_{v}+M\right) \sqrt{ } 5}
$$

Differentiation of the extreme right hand member with respect to $N_{v}$ shows 
that it is a decreasing function provided $N_{v}<\left(2 h_{M}^{2} / v^{2} M\right)$. Now from the definition of $h_{M}$, it follows that

$$
\min _{M}\left(2 h_{M}^{2} / v^{2} M\right)>8 \cdot 10^{5},
$$

with the minimum occurring when $M=10$. Hence for all $M$, the function $\left(h_{M}^{2}+v^{2} N_{v}^{2}\right)^{1 / 2} /\left(2 N_{v}+M\right)$ is decreasing in $N_{v}$ so that its minimum occurs at $N_{v}=223,606$. Since for this value of $N_{v}$ the value of $v N_{v}>1$, this minimum is greater than

$$
\frac{\left(h_{M}^{2}+1\right)^{1 / 2}}{447,212+M} \geqq \frac{(447,212+M) \sqrt{ } 5 \cdot 10^{-6}}{447,212+M} \geqq \sqrt{ } 5 \cdot 10^{-6} .
$$

(Note that except for the last four values of $M$,

$$
\left.\left(h_{M}^{2}+1\right)^{1 / 2} \geqq(447,212+2 M) \sqrt{ } 5 \cdot 10^{-6} .\right)
$$

Hence $r(X) \geqq .9932$.

Case 3. $X$ meets row $\alpha$, row $\beta$ and one of the columns $\gamma$ or $\delta$. In this case $d>1-2 \cdot 10^{-6}$ and therefore if $N \leqq 447,212$ then $r(X) \geqq 1$. In the remainder of this case, we shall take $N>447,212$, where $N$ denotes the total number of rectangles $R_{i}$ that intersect $X$ nonvacuously.

Let $M+24$ be the iargest value and $m$ be the smallest value of $n \leqq 276,394$ such that $R_{2 n} \cap X \neq \emptyset$; let $K+24$ be the largest value and $k$ be the smallest value of $n \leqq 276,394$ such that $R_{2 n-1} \cap X \neq \emptyset$. We may take $M \geqq 10, K \geqq 10$ for otherwise an argument like Case 2 above shows that $r(X)>.999$. Note that $d>h_{M}, d>h_{K}$ and no rectangle from either column $\gamma$ or $\delta$ can meet $X$ above the line

$$
y=\min \left(\left(d^{2}-h_{M}^{2}\right)^{1 / 2}+10^{-6}, 1\right)
$$

nor below the line

$$
y=\max \left(1-\left(d^{2}-h_{K}^{2}\right)^{1 / 2}-10^{-6}, 0\right) .
$$

Now we temporarily assume that

$$
h_{M} \geqq\left(d^{2}-\left(1-2 \cdot 10^{-6}\right)^{2}\right)^{1 / 2} \text { and } h_{K} \geqq\left(d^{2}-\left(1-2 \cdot 10^{-6}\right)^{2}\right)^{1 / 2} .
$$

Then the number of rectangles from columns $\gamma$ and $\delta$ that meet $X$ is no larger than

$$
\begin{aligned}
&\left.2\left(\left(\left(d^{2}-h_{M}^{2}\right)^{1 / 2}+\left(d^{2}-h_{K}^{2}\right)^{1 / 2}-1+2 \cdot 10^{-6}\right) / v\right)+2\right) \\
& \leqq 2 \sqrt{ } 5 \cdot 10^{5}\left(\left(d^{2}-h_{M}^{2}\right)^{1 / 2}+\left(d^{2}-h_{K}^{2}\right)^{1 / 2}-1\right)+6 .
\end{aligned}
$$

Also

$$
\begin{aligned}
& h_{k+9} \geqq h_{M}-\left(d^{2}-\left(1-2 \cdot 10^{-6}\right)^{2}\right)^{1 / 2}, \\
& h_{m+9} \geqq h_{K}-\left(d^{2}-\left(1-2 \cdot 10^{-6}\right)^{2}\right)^{1 / 2} .
\end{aligned}
$$


Then

$$
\begin{gathered}
(447,212+2(k+9))^{2} \cdot 5 \cdot 10^{-12}-1=h_{k+9}^{2} \geqq\left(h_{M}-\left(d^{2}-\left(1-2 \cdot 10^{-6}\right)^{2}\right)^{1 / 2}\right)^{2}, \\
k+9 \geqq \sqrt{ } 5 \cdot 10^{5}\left(1+\left(h_{M}-\left(d^{2}-\left(1-2 \cdot 10^{-6}\right)^{2}\right)^{1 / 2}\right)^{2}\right)^{1 / 2}-223,606,
\end{gathered}
$$

and similarly

$$
m+9 \geqq \sqrt{ } 5 \cdot 10^{5}\left(1+\left(h_{K}-\left(d^{2}-\left(1-2 \cdot 10^{-6}\right)^{2}\right)^{1 / 2}\right)^{2}\right)^{1 / 2}-223,606 .
$$

Combining the above, it follows that $N \leqq g^{*}\left(d, h_{M}, h_{K}\right)$ where

$$
\begin{aligned}
g^{*}\left(d, h_{M}, h_{K}\right)= & 2 \sqrt{ } 5 \cdot 10^{5}\left(\left(d^{2}-h_{M}^{2}\right)^{1 / 2}+\left(d^{2}-h_{K}^{2}\right)^{1 / 2}-1\right)+6 \\
& +K+25-\sqrt{ } 5 \cdot 10^{5}\left(1+\left(h_{M}-\left(d^{2}-\left(1-2 \cdot 10^{-6}\right)^{2}\right)^{1 / 2}\right)^{2}\right)^{1 / 2} \\
& +223,606+9+M+25 \\
& -\sqrt{ } 5 \cdot 10^{5}\left(1+\left(h_{K}-\left(d^{2}-\left(1-2 \cdot 10^{-6}\right)^{2}\right)^{1 / 2}\right)^{2}\right)^{1 / 2} \\
& +223,606+9 \\
= & \sqrt{ } 5 \cdot 10^{5}\left(2\left(d^{2}-h_{M}^{2}\right)^{1 / 2}+2\left(d^{2}-h_{K}^{2}\right)^{1 / 2}-2\right. \\
& +\left(1+h_{M}^{2}\right)^{1 / 2}+\left(1+h_{K}^{2}\right)^{1 / 2} \\
& -\left(1+\left(h_{M}-\left(d^{2}-\left(1-2 \cdot 10^{-6}\right)^{2}\right)^{1 / 2}\right)^{2}\right)^{1 / 2} \\
& \left.-\left(1+\left(h_{K}-\left(d^{2}-\left(1-2 \cdot 10^{-6}\right)^{2}\right)^{1 / 2}\right)^{2}\right)^{1 / 2}\right) \\
& +74 .
\end{aligned}
$$

To maximize $g^{*}$ holding $d$ fixed, note that

$$
\begin{aligned}
\frac{\partial g^{*}}{\partial h_{M}}=\sqrt{ } 5 \cdot 10^{5}\left(\frac{h_{M}}{\left(1+h_{M}^{2}\right)^{1 / 2}}-\frac{2 h_{M}}{\left(d^{2}-h_{M}^{2}\right)^{1 / 2}}\right. & \left.-\frac{\left(h_{M}-\left(d^{2}-\left(1-2 \cdot 10^{-6}\right)^{2}\right)^{1 / 2}\right.}{\left(1+\left(h_{M}-\left(d^{2}-\left(1-2 \cdot 10^{-6}\right)^{2}\right)^{1 / 2}\right)^{2}\right)^{1 / 2}}\right) .
\end{aligned}
$$

Then $\partial g^{*} / \partial h_{M}<0$ under the assumption $\left({ }^{*}\right)$, for then $\left(d^{2}-h_{M}^{2}\right)^{1 / 2}<1$. Hence we need only evaluate $g^{*}$ at the smallest integral value of $M \geqq 10$ for which $h_{M} \geqq\left(d^{2}-\left(1-2 \cdot 10^{-6}\right)^{2}\right)^{1 / 2}$; similarly for $K$, so that $K=M$. If $K=M=10$, then

$$
\begin{aligned}
g^{*}= & \sqrt{ } 5 \cdot 10^{5}\left(4\left(d^{2}-h_{10}^{2}\right)^{1 / 2}-2-2\left(1+\left(h_{10}-\left(d^{2}-\left(1-2 \cdot 10^{-6}\right)^{2}\right)^{1 / 2}\right)^{2}\right)^{1 / 2}\right) \\
& +447,212+94 \\
\leqq & 447,212+94<2 \sqrt{ } 5 \cdot 10^{5}+94
\end{aligned}
$$

so that

$$
r(X) \geqq \frac{d \cdot 10^{6}}{\sqrt{ } 5 \cdot N}>\frac{\left(1-2 \cdot 10^{-6}\right) \cdot 10^{6}}{\sqrt{ } 5\left(2 \sqrt{ } 5 \cdot 10^{5}+94\right)}=\frac{10^{6}-2}{10^{6}+94 \sqrt{ } 5}>0.999 .
$$

If $K=M>10$, then $h_{M-1}<\left(d^{2}-\left(1-2 \cdot 10^{-6}\right)^{2}\right)^{1 / 2}$, and so 


$$
\begin{aligned}
g^{*}= & 2 \sqrt{ } 5 \cdot 10^{5}\left(2\left(d^{2}-h_{M}^{2}\right)^{1 / 2}-1-\left(1+\left(h_{M}-\left(d^{2}-\left(1-2 \cdot 10^{-6}\right)^{2}\right)^{1 / 2}\right)^{2}\right)^{1 / 2}\right) \\
& +2(M-1)+447,212+76 \\
= & 2 \sqrt{ } 5 \cdot 10^{5}\left(2\left(d^{2}-h_{M}^{2}\right)^{1 / 2}-1-\left(1+\left(h_{M}-\left(d^{2}-\left(1-2 \cdot 10^{-6}\right)^{2}\right)^{1 / 2}\right)^{2}\right)^{1 / 2}\right) \\
& \left.+\left(1+h_{M-1}^{2}\right)^{1 / 2}\right)+76 \\
< & 2 \sqrt{ } 5 \cdot 10^{5}\left(1+h_{M-1}^{2}\right)^{1 / 2}+76 \\
< & 2 \sqrt{ } 5 \cdot 10^{5}\left(1+d^{2}-\left(1-2 \cdot 10^{-6}\right)^{2}\right)^{1 / 2}+76 \\
< & 2 \sqrt{ } 5 \cdot 10^{5}\left(d^{2}+4 \cdot 10^{-6}\right)^{1 / 2}+76 \\
< & 2 \sqrt{ } 5 \cdot 10^{5}\left(d+3 \cdot 10^{-6}\right)+76
\end{aligned}
$$

hence

$$
r(X) \geqq \frac{d \cdot 10^{6}}{\sqrt{ } 5 \cdot N}>\frac{10^{6} \cdot d}{10^{6} \cdot d+3+76 \sqrt{ } 5}>0.999 .
$$

If instead of assuming $\left({ }^{*}\right)$, we had assumed say

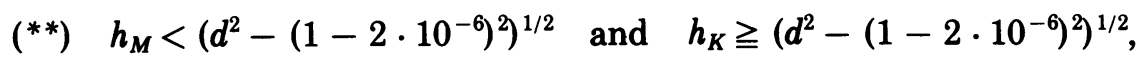

then the previous argument suitably modified gives $N \leqq g^{* *}\left(d, h_{M}, h_{K}\right)$ where

$$
\begin{aligned}
g^{* *}\left(d, h_{M}, h_{K}\right)= & 2 \sqrt{ } 5 \cdot 10^{5}\left(\left(d^{2}-h_{K}^{2}\right)^{1 / 2}\right)+4+K+24+M+25 \\
& -\sqrt{ } 5 \cdot 10^{5}\left(1+\left(h_{K}-\left(d^{2}-\left(1-2 \cdot 10^{-6}\right)^{2}\right)^{1 / 2}\right)^{2}\right)^{1 / 2} \\
& +223,606+9 \\
= & \sqrt{ } 5 \cdot 10^{5}\left(2\left(d^{2}-h_{K}^{2}\right)^{1 / 2}+\left(1+h_{K}^{2}\right)^{1 / 2}\right. \\
& \left.-\left(1+\left(h_{K}-\left(d^{2}-\left(1-2 \cdot 10^{-6}\right)^{2}\right)^{1 / 2}\right)^{2}\right)^{1 / 2}\right)+M+62 .
\end{aligned}
$$

Then $\partial g^{* *} / \partial h_{K}<0$, so long as $h_{K} \geqq\left(d^{2}-\left(1-2 \cdot 10^{-6}\right)^{2}\right)^{1 / 2}$. Hence we evaluate $g^{* *}$ at the smallest value of $K>10$ for which

$$
h_{K} \geqq\left(d^{2}-\left(1-2 \cdot 10^{-6}\right)^{2}\right)^{1 / 2} \text {. }
$$

At this value of $K$,

$$
\begin{aligned}
g^{* *} & <\sqrt{ } 5 \cdot 10^{5}+K+M+223,606+62 \\
& <223,606+(K-1)+223,606+M+64 \\
& =\sqrt{ } 5 \cdot 10^{5}\left(\left(1+h_{K-1}^{2}\right)^{1 / 2}+\left(1+h_{M}^{2}\right)^{1 / 2}\right)+64 \\
& <2 \sqrt{ } 5 \cdot 10^{5}\left(\left(1+d^{2}-\left(1-2 \cdot 10^{-6}\right)^{2}\right)^{1 / 2}\right)+64 \\
& <2 \sqrt{ } 5 \cdot 10^{5}\left(d+3 \cdot 10^{-6}\right)+64,
\end{aligned}
$$

so that

$$
r(X) \geqq \frac{d \cdot 10^{6}}{\sqrt{5} \cdot N}>\frac{d \cdot 10^{6}}{10^{6}\left(d+3 \cdot 10^{-6}\right)+64 \sqrt{ } 5}>0.999
$$

Finally, if we assume 
$\left.{ }^{* * *}\right) h_{M}<\left(d^{2}-\left(1-2 \cdot 10^{-6}\right)^{2}\right)^{1 / 2}$ and $h_{K}<\left(d^{2}-\left(1-2 \cdot 10^{-6}\right)^{2}\right)^{1 / 2}$,

then

$$
\begin{aligned}
N & \leqq 447,212+M+24+K+24 \\
& =\sqrt{ } 5 \cdot 10^{5}\left(\left(1+h_{M}^{2}\right)^{1 / 2}+\left(1+h_{K}^{2}\right)^{1 / 2}\right)+48 \\
& <2 \sqrt{ } 5 \cdot 10^{5}\left(1+d^{2}-\left(1-2 \cdot 10^{-6}\right)^{2}\right)^{1 / 2}+48 \\
& <2 \sqrt{ } 5 \cdot 10^{5}\left(d+3 \cdot 10^{-6}\right)+48
\end{aligned}
$$

so that

$$
r(X) \geqq \frac{d \cdot 10^{6}}{\sqrt{ } 5 \cdot N}>\frac{d \cdot 10^{6}}{10^{6}\left(d+3 \cdot 10^{-6}\right)+48 \sqrt{ } 5}>0.999 .
$$

Theorem 5.5. 2.2207 $<0.9932 \sqrt{ } 5 \leqq C_{2}^{1}(A)$.

Proof. Let $X_{1}, X_{2}, \cdots$ be a covering of $A$ by nonvacuous open convex sets. Since $A$ is compact, we may assume that $A \subset \bigcup_{n=1}^{k} X_{n}$. Let $0<\lambda<1$ be arbitrary. Using Theorem 5.3, choose the integer $i$ so that

$$
\frac{d_{n} \cdot 10^{6 i}}{N_{n, i} \sqrt{5}}>\min \left(\lambda \cdot r\left(X_{n}\right), 1\right) \text { for } n=1, \cdots, k,
$$

where $d_{n}=\operatorname{diam} X_{n}$ and $N_{n, i}$ is the number of rectangles of $A_{i}$ with nonvacuous intersection with $X_{n}$. By Theorem 5.4, $r\left(X_{n}\right) \geqq 0.9932$, so that for $n=1, \cdots, k$,

$$
d_{n}>0.9932 \lambda N_{n, i} \sqrt{ } 5 / 10^{6 i} .
$$

Since $A \subset \bigcup_{n=1}^{k} X_{n}$, it follows that $\sum_{n=1}^{k} N_{n, i} \geqq 10^{6 i}$, and hence

$$
\sum_{n=1}^{\infty} d_{n} \geqq \sum_{n=1}^{k} d_{n}>0.9932 \lambda \sqrt{ } 5 \cdot \sum_{n=1}^{k} N_{n, i} / 10^{6 i} \geqq 0.9932 \lambda \sqrt{ } 5 .
$$

Hence $C_{2}^{1}(A) \geqq 0.9932 \lambda \sqrt{ } 5$, and letting $\lambda \rightarrow 1$,

$$
C_{2}^{1}(A) \geqq 0.9932 \sqrt{ } 5 \text {. }
$$

\section{The Carathéodory 2-measure of $A \times[0,1]$.}

Theorem 6.1. $C_{3}^{2}(A \times[0,1])<2.1715$.

Proof. Using the notation of Definition 4.2, we shall first cover $R \times[0,1]$ by a finite number of open convex sets, where $R=A_{0}^{*}$ (see Definition 4.3 for $\left.A_{0}^{*}\right)$. For this purpose let $\epsilon>0$ and define the open convex set $U_{0}$ by

$$
\begin{array}{r}
U_{0}=\left\{(x, y, z) \mid-\epsilon<y<1+\epsilon,-\epsilon<x \leqq 104 \cdot 10^{-6},-\epsilon<z<1+\epsilon\right\} \\
\cup\left\{(x, y, z) \mid-\epsilon<y<1+\epsilon, 104 \cdot 10^{-6}<x<2+2 \epsilon,\right. \\
\left.-\epsilon<z<1+\epsilon-\frac{(1+2 \epsilon)\left(x-104 \cdot 10^{-6}\right)}{2+2 \epsilon-104 \cdot 10^{-6}}\right\} .
\end{array}
$$


For $n=1$ through 276,394, define the open convex sets $U_{2 n}$ and $U_{2 n-1}$ by

$$
\begin{array}{r}
U_{2 n}=\left\{(x, y, z) \mid h_{n}-\epsilon<x<h_{n}+2 \cdot 10^{-6}+\epsilon,-\epsilon / 2<y<10^{-6}+\epsilon / 2,\right. \\
\left.1-\frac{1}{2}\left(h_{n}+2 \cdot 10^{-6}\right)<z<1+\epsilon\right\}, \\
U_{2 n-1}=\left\{(x, y, z) \mid h_{n}-\epsilon<x<h_{n}+2 \cdot 10^{-6}+\epsilon, 1-10^{-6}-\epsilon / 2<y<1+\epsilon / 2,\right. \\
\left.1-\frac{1}{2}\left(h_{n}+2 \cdot 10^{-6}\right)<z<1+\epsilon\right\} .
\end{array}
$$

Then the 552,789 sets $U_{i}$ cover $R \times[0,1]$. If $g_{2}$ is as defined in 3.1 , then it is simple to check that

$$
g_{2}\left(U_{0}\right)=(1+2 \epsilon)\left((1+2 \epsilon)^{2}+\left(1+2 \epsilon+52 \cdot 10^{-6}\right)^{2}\right)^{1 / 2},
$$

since the area of the projection of $U_{0}$ in the $y$-axis direction is $(1+2 \epsilon)$ $\times\left(1+2 \epsilon+52 \cdot 10^{-6}\right)$ while the projected area in the $x$-axis direction is $(1+2 \epsilon)^{2}$. Similarly one can check that

$$
g_{2}\left(U_{2 n}\right)=g_{2}\left(U_{2 n-1}\right)=\sqrt{ } 5\left(10^{-6}+\epsilon\right)\left(\epsilon+10^{-6}+\frac{1}{2} h_{n}\right) .
$$

To estimate $\sum_{i} g_{2}\left(U_{i}\right)$, it is obvious that one must first estimate $\sum_{i} h_{i}$, which we now do. Thus

$$
\begin{aligned}
\sum_{i=1}^{276,394} h_{i} & =\sum_{i=1}^{9} h_{i}+\sum_{i=10}^{276,390} h_{i}+\sum_{i=276,391}^{276,394} h_{i} \\
& <9(9049.5) \cdot 10^{-6}+\int_{10}^{276,394}\left((447,212+2 x)^{2} 5 \cdot 10^{-12}-1\right)^{1 / 2} d x \\
& <82 \cdot 10^{-3}+\frac{1}{2} \sqrt{ } 5 \cdot 10^{5}(2 \sqrt{ } 5-\ln (2+\sqrt{ } 5)) .
\end{aligned}
$$

Combining the above calculations yields

$$
\begin{aligned}
\sum_{i=0}^{552,788} g_{2}\left(U_{i}\right)< & (1+2 \epsilon)\left((1+2 \epsilon)^{2}+\left(1+2 \epsilon+52 \cdot 10^{-6}\right)^{2}\right)^{1 / 2} \\
& +\sqrt{ } 5\left(10^{-6}+\epsilon\right)^{2} \cdot 552,788 \\
& +\sqrt{ } 5\left(10^{-6}+\epsilon\right)\left(82 \cdot 10^{-3}+\frac{1}{2} \sqrt{ } 5 \cdot 10^{5}(2 \sqrt{ } 5-\ln (2+\sqrt{ } 5))\right) \\
< & \sqrt{ } 2(1+2 \epsilon)\left(1+2 \epsilon+52 \cdot 10^{-6}\right)+\sqrt{ } 5\left(10^{-6}+\epsilon\right) \\
& \cdot\left(.635+6 \cdot 10^{5} \epsilon+\frac{1}{2} \sqrt{ } 5 \cdot 10^{5}(2 \sqrt{ } 5-\ln (2+\sqrt{ } 5))\right) .
\end{aligned}
$$

If now $\epsilon$ is restricted to be less than $10^{-6}$, then it is obvious that each $U_{i} \in \mathscr{M}_{1 / 2}$ (see Definition 3.2).

Recalling the construction of $A$, we see that at the $i$ th stage of construction, $10^{12 i}$ copies of the collection of sets $\left\{U_{i}\right\}$, each set being scaled down in the ratio $10^{-6 i}$, will suffice to cover $A \times[0,1]$. The reason for this is that in $A_{i}$ there are $10^{6 i}$ rectangles, and to cover the cylinder of unit height above one of these rectangles it is sufficient to pile $10^{6 i}$ copies of the scaled down $\left\{U_{i}\right\}$ one on top of the other. Observe that scaling down a set $U_{i}$ in the ratio 
$10^{-6 i}$ has the effect of multiplying $g_{2}\left(U_{i}\right)$ by $10^{-12 i}$, so that at the $i$ th stage the sum of $g_{2}$ values of the covering sets (each in $\mathscr{M}_{1 / 2}$ ) is independent of $i$. Letting $\epsilon \rightarrow 0$ and applying Theorem 3.8, we obtain

$$
\begin{aligned}
D_{1 / 2}(A \times[0,1])= & C_{3}^{2}(A \times[0,1]) \\
\leq & \sqrt{ } 2\left(1+52 \cdot 10^{-6}\right) \\
& +\sqrt{ } 5 \cdot 10^{-6}\left(\frac{1}{2} \sqrt{ } 5 \cdot 10^{5}(2 \sqrt{ } 5-\ln (2+\sqrt{ } 5))+.635\right) \\
< & 2.1715 .
\end{aligned}
$$

REMARK 6.2. By refining the constructions and arguments in $\$ 5$ and 6 , one can decrease the ratio of $C_{3}^{2}(A \times[0,1]) / C_{2}^{1}(A)$. Thus if in the construction of $A$, one uses more rectangles at each stage of construction, one can force $C_{2}^{1}(A)$ to equal $\sqrt{ } 5$. If in addition, in Theorem 6.1, one uses more sets of the form $U_{0}$ instead of the cylinders $U_{i}(i>0)$, one can obtain

$$
C_{3}^{2}(A \times[0,1]) \leqq 4 \sqrt{ } 10 /(2 \sqrt{ } 5+\ln (2+\sqrt{ } 5)),
$$

so that

$$
C_{3}^{2}(A \times[0,1]) / C_{2}^{1}(A) \leqq 4 \sqrt{ } 2 /(2 \sqrt{ } 5+\ln (2+\sqrt{ } 5)) \approx 0.957 .
$$

Moreover, if one varies the ratio of the sides of the rectangles used so that instead of 2:1 the ratio is $a: 1$, then the analogous construction yields

$$
C_{3}^{2}(A \times[0,1]) / C_{2}^{1}(A) \leqq a\left(4+a^{2}\right)^{1 / 2} /\left(a\left(1+a^{2}\right)^{1 / 2}+\ln \left(a+\left(1+a^{2}\right)^{1 / 2}\right)\right) .
$$

The minimum of the right hand member is achieved at a value of $a \approx 2.4$ and this minimum value is approximately $.9552-$.

Thus, if

$$
\rho=\inf _{0<C_{2}^{1}(X)<\infty} C_{3}^{2}(X \times[0,1]) / C_{2}^{1}(X),
$$

the preceding paragraph taken in conjunction with the results of [3], implies $\frac{1}{2} \leqq \rho<.9552$. A sharpening of the bounds on $\rho$ would be interesting.

\section{BiBLIOGRAPHY}

1. A. S. Besicovitch and P. A. P. Moran, The measure of product and cylinder sets, J. London Math. Soc. 20 (1945), 110-120.

2. G. Freilich, On the measure of cartesian product sets, Trans. Amer. Math. Soc. 69 (1950), 232-275.

3. _ Two-dimensional measure in 3-space, Proc. Amer. Math. Soc. 6 (1955), 631-633.

4. A. P. Morse and J. F. Randolph, Gillespie measure, Duke Math. J. 6 (1940), 408-419.

5. J. F. Randolph, On generalizations of length and area, Bull. Amer. Math. Soc. 42 (1936), 268-274.

The City College,

New York, New YoRK 